\title{
Aprendizaje basado en proyectos: Primera experiencia en la asignatura de Física del Grado en Ingeniería de Telecomunicación, Sonido e Imagen.
}

\author{
Jesús Alba Fernández ${ }^{a}$, Constantino Torregrosa Cabanilles ${ }^{\mathrm{a}}$ y Romina del Rey \\ Tormos $^{\mathrm{a}}$ \\ aUniversitat Politècnica de Valéncia, Escuela Politécnica Superior de Gandia, Departamento de Física \\ Aplicada, C/Paraninfo nº - 46715 Grao de Gandia. jesalba@fis.upv.es, ctorregr@fis.upv.es, \\ roderey@doctor.upv.es
}

\begin{abstract}
This paper presents an implementation of the project based learning methods to Introductory Physics courses of the Telecommunication, Sound and Image Engineering Degree. This first project starts at the last lessons of the subject, devoted to magnetic field and electromagnetic induction.

It has been organized in terms of practical projects that the students can choose voluntarily. Here we show how this active methodology was organized, from the context, aims, previous questions, procedures, planning, types of activities, working groups, until the evaluation and conclusions.
\end{abstract}

Keywords: Project Based Learning, electromagnetism, electromagnetic induction, active methodologies

\begin{abstract}
Resumen
Esta comunicación trata sobre la prueba de aplicación del aprendizaje basado en proyectos en la asignatura de Física del Grado en Ingeniería de Telecomunicación, Sonido e Imagen. Se realiza esta primera experiencia donde el último bloque temático dedicado al campo magnético y a la inducción magnética se articula en forma de proyectos que los alumnos eligen de forma voluntaria. En la comunicación se resumen cómo se ha articulo esta metodología activa, desde el contexto, objetivos, preguntas motrices, enunciado, planificación, tipos de actividades, interdependencia, valoración y conclusiones.
\end{abstract}

Palabras clave: Aprendizaje Basado en Proyectos, campo eléctrico, inducción electromagnética, metodologías activas 
Aprendizaje basado en proyectos: Primera experiencia en la asignatura de Física del Grado en Ingeniería de Telecomunicación, Sonido e Imagen

\section{Introducción}

Transformar el conocimiento en algo práctico es un concepto que puede definir la ingeniería. Los conocimientos científicos se aplican para ingeniar o perfeccionar técnicas, con un tiempo limitado, en base a los recursos disponibles, coste, estética, etc. (Wright y Samaniego, 2004; Aparicio et al., 2005).

Para el caso de ingeniería la estrategia de enseñanza activa, el Aprendizaje Basado en Proyectos (ABP o PBL, Project-based learning), parece muy adecuado ya que se realiza un proyecto, normalmente de cierta envergadura y en grupo. Este proyecto debe previamente analizarse por el profesor o profesores y asegurarse de que los alumnos disponen de todo lo que hace falta para resolverlo, y que en su resolución desarrollará todas las destrezas que se desean. Este método docente hace que el estudiante sea protagonista de su propio aprendizaje. Este tipo de estrategia puede ayudar a que los futuros ingenieros se acerquen a un proyecto real de ingeniería antes de finalizar su carrera (Case y Light, 2011).

En general, en la técnica de PBL los alumnos se agrupan en proyectos relativamente abiertos y en muchos casos multidisciplinares buscando simular entornos profesionales en los que aplicar ciertos conocimientos y habilidades. Gracias a esta técnica deben manejar diferentes fuentes de información, analizar y sintetizar, resolver problemas complejos que abarcan varias disciplinas, pensamiento crítico, planifcarse, organizarse, tomar decisiones, etc (De Miguel, 2006). Se abordan proyectos que puedan implementarse. Existen múltiples ejemplos al respecto (Urraza y Ortega, 2009) (Calvo et al, 2010) (Gonzalez-Jorge et al, 2014), (Alba et al, 2015).

En este trabajo se realizan propuestas de PBLs que involucra a la asignatura FISICA del Grado en Ingeniería de Sistemas de Telecomunicaciones, Sonido e Imagen (GISTSI). Hay que tener en cuenta que en el caso de estas asignaturas los alumnos ya parten de una acepción negativa puesto que se presupone que las asignaturas básicas son más bien teóricas y tienen menos conexión con el mundo profesional al que deberá incorporarse el alumno al finalizar sus estudios. Esto produce cierta desmotivación que debe corregirse.

La asignatura FISICA es una asignatura anual fundamental de primer curso. En el segundo semestre se tratan los siguientes temas:

- Campo eléctrico

- Potencial eléctrico

- Corriente eléctrica

- Campo magnético

- Inducción electromagnética 
Estos cinco bloques temáticos deben impartirse en 15 semanas de clase en el segundo semestre. Además realizan 4 prácticas de 2 horas durante el dicho semestre. Por tanto, el alumno tiene 45 horas presenciales en el citado semestre y 67,5 no presenciales realizando el cómputo de horas según créditos.

En el PBL propuesto, se abordan los dos últimos temas, aunque también son necesarios conocimientos de los temas anteriores (Serway y Jewett, 2005, 2008; Tipler 1992, 1994).

\section{Objetivos}

Se plantea como objetivo la incorporación progresiva de PBLs en la asignatura de física del GISTSI. Estos PBLs se implementarán prácticamente en las últimas 6 semanas de clase, aunque de forma efectiva en el último mes. Se realizan una serie de modificaciones respecto a lo que es habitual. Los alumnos pueden elegir o no el realizar un proyecto, es decir, es voluntario. Los que no realizan el proyecto siguen el proceso de evaluación clásico de la asignatura. Los que deciden realizar el proyecto pueden elegir entre un banco de proyectos a desarrollar o, incluso, proponer algún proyecto que no esté en el listado. Puede haber distintos grupos trabajando en un mismo proyecto. A partir de ahí, cada grupo sigue la metodología clásica de un PBL que se describirá a continuación en el siguiente punto.

La idea fundamental es la incorporación progresiva de PBLs pero sin perjudicar a los profesores, ya que se han dado casos en los que los alumnos valoran negativamente el realizar este tipo de experiencias de forma obligatoria. Al hacerse de forma voluntaria, los alumnos están más motivados. Al dar también la opción de elegir el tema, los alumnos que lo desean pueden trabajar más cómodamente y el profesor también puede valorar el grado de implicación de los alumnos. También puede verse el porcentaje de alumnos reacios a cualquier cambio por diferentes motivos. Obviamente, la carga de trabajo para los profesores es mayor.

\section{Desarrollo de la innovación}

En este apartado se realiza la descripción la innovación, teniendo en cuenta que para el desarrollo del PBL se pueden elegir diferentes proyectos.

Algúnos títulos de los proyectos, pregunta motriz y ejemplos.

- Fabricación de un motor de bajo coste: Si a un motor eléctrico se le alimenta con corriente continua ¿por qué giran algunas de sus piezas? https://www.youtube.com/watch?v=-KzK7W86Y7E

- Construcción de un altavoz dinámico. ¿cómo es posible que suene un altavoz? https://www.youtube.com/watch?v=e4qxNopUZYM 
Aprendizaje basado en proyectos: Primera experiencia en la asignatura de Física del Grado en Ingeniería de Telecomunicación, Sonido e Imagen

- Construcción de un mini cañon acústico. ¿se puede convertir un altavoz en un arma? https://www.youtube.com/watch?v=Ft21O7NelY4

- Visualización de campos magnéticos en 3D. https://www.youtube.com/watch?v=Y7CPYM3IuDA

- Diseño de un Levitron. ¿Por qué flota el tren japonés? https://www.youtube.com/watch?v=316nJTkhBPs\&index=2\&list=PLoAGB6mBw smVLTKqLciMg8tVqmhHsm_jO

Los objetivos comunes son los siguientes. Al finalizar el proyecto, el estudiante será capaz de:

- Explicar el concepto de campo magnético y fuerza magnética

- Plantear problemas con fuerzas y campos magnéticos

- Dibujar las líneas de campo magnético en cualquier caso

- Explicar y plantear problemas basados en Lenz y Faraday

Las actividades propuestas son las siguientes:

- Búsqueda de información

- Lectura de materiales complementarios

- Test individual de comprensión de conceptos por asignatura

- Exposición pública en clase de los proyectos

El plan de trabajo general se simplifica en la siguiente tabla (la última planificación realizada)

Tabla 1.

\begin{tabular}{|l|l|l|}
\cline { 2 - 3 } \multicolumn{1}{c|}{} & SEMANA & TAREAS \\
\hline Semana 1 & $20 / 04 / 15-26 / 04 / 15$ & Búsqueda de información. Lectura de materiales \\
\hline Semana 2 & $27 / 04 / 15-03 / 05 / 15$ & Trabajo en grupo. \\
\hline Semana 3 & $04 / 05 / 15-10 / 05 / 15$ & Trabajo en grupo. Entregable 1. \\
\hline Semana 4 & $11 / 05 / 15-17 / 05 / 15$ & Trabajo en grupo. \\
\hline Semana 5 & $18 / 05 / 15-24 / 05 / 15$ & Trabajo en grupo. Problema 9 \\
\hline Semana 6 & $25 / 05 / 15-31 / 05 / 15$ & Entregable 2. Presentación del trabajo \\
\hline Semana 7 & $01 / 06 / 15-07 / 06 / 15$ & Problema 10. \\
\hline
\end{tabular}

Entregable 1. 07/05/15. Entrega de la descripción del posible prototipo y materiales a utilizar. Previsión de cálculos teóricos del prototipo.

Problema 9. 21/05/15. Problema del tema 11 según planificación de clase.

Entregable 2. 28/05/15. Entrega/demostración del prototipo en clase. Entrega de un poster explicativo en tamaño A2. Los alumnos incluirán en la entrega qué porcentaje ha trabajado cada miembro del grupo en el proyecto.

Problema 10. 04/06/15. Problema del tema 12 según planificación de clase. 
Todos los entregables son de grupo.

La valoración final se compone de tres tipos de evaluaciones

1) La evaluación continua del alumno con reuniones periódicas con los profesores tutores, verificando el cumplimiento de tiempos de ejecución y resultados de cada fase. Se analizan aspectos como responsabilidad personal, iniciativa, grado de implicación y toma de decisiones

2) Evaluación centrada en cada asignatura a través de dos problemas de los temas correspondientes

3) Evaluación del grado de consecución de las competencias propuestas a través de dos rúbricas, una para que los propios alumnos evalúen (tabla 2) y otra para los profesores (tabla 3). Ambas se ponderan para obtener la nota final.

Además se valora la interdependencia positiva y la exigibilidad personal a través de un test individual y preguntas en la exposición. Se subirá un punto a los miembros del grupo en el caso de que todos ellos obtengan una calificación superior a 6 .

Tabla 2. Rúbrica para alumnos

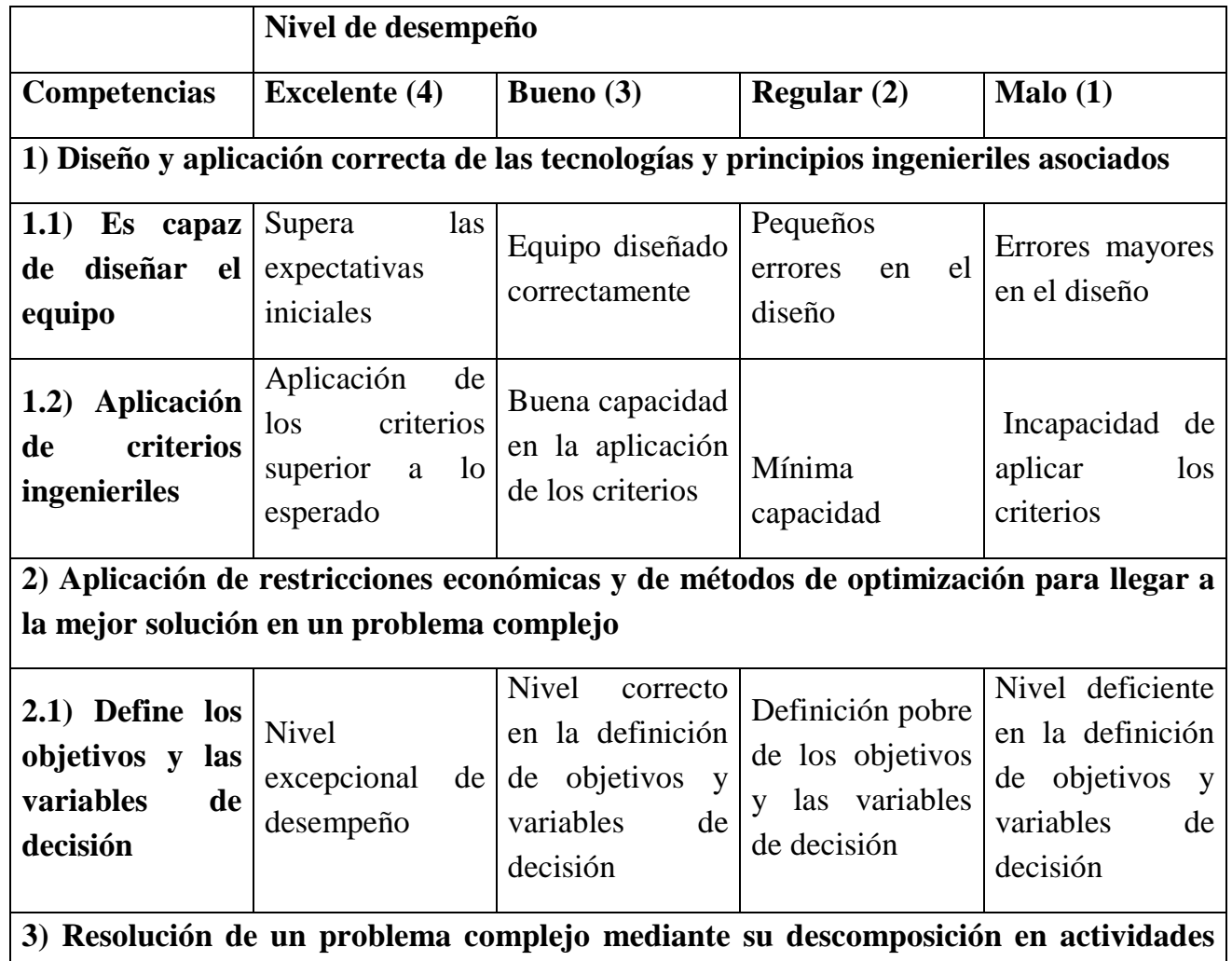

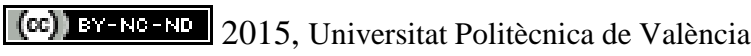

Congreso In-Red (2015) 
Aprendizaje basado en proyectos: Primera experiencia en la asignatura de Física del Grado en Ingeniería de Telecomunicación, Sonido e Imagen

\begin{tabular}{|c|c|c|c|c|}
\hline $\begin{array}{l}\text { 3.1) Reconoce } \\
\text { problemas más } \\
\text { sencillos } \\
\text { integrados en el } \\
\text { problema } \\
\text { global }\end{array}$ & $\begin{array}{l}\text { Capacidad } \\
\text { superior para } \\
\text { reconocer los } \\
\text { componentes del } \\
\text { problema global }\end{array}$ & $\begin{array}{l}\text { Buena capacidad } \\
\text { para } \\
\text { descomponer un } \\
\text { problema en sus } \\
\text { partes }\end{array}$ & $\begin{array}{l}\text { Poca capacidad } \\
\text { para } \\
\text { descomponer los } \\
\text { componentes del } \\
\text { problema }\end{array}$ & $\begin{array}{l}\text { Incapacidad } \\
\text { para reconocer } \\
\text { los componentes } \\
\text { del problema }\end{array}$ \\
\hline \multicolumn{5}{|c|}{ 4) Suficiencia en la comunicación oral y escrita de sus ideas y trabajos realizados } \\
\hline $\begin{array}{l}\text { 4.1) Mecánica } \\
\text { de la } \\
\text { presentación }\end{array}$ & $\begin{array}{ll}\text { Confiado } & \text { y } \\
\text { claridad en la } \\
\text { exposición }\end{array}$ & $\begin{array}{l}\text { Buena } \\
\text { presentación }\end{array}$ & $\begin{array}{l}\text { Algo nervioso, } \\
\text { actitud } \\
\text { ligeramente } \\
\text { insegura }\end{array}$ & $\begin{array}{l}\text { Muy nervioso e } \\
\text { inseguro }\end{array}$ \\
\hline 4.2) Respuestas & $\begin{array}{l}\text { Respuestas } \\
\text { correctas y con } \\
\text { matices }\end{array}$ & $\begin{array}{l}\text { Respuestas } \\
\text { correctas }\end{array}$ & $\begin{array}{l}\text { Alguna } \\
\text { respuesta } \\
\text { incorrecta }\end{array}$ & $\begin{array}{l}\text { No responde o } \\
\text { con evasivas }\end{array}$ \\
\hline 4.3) Póster & $\begin{array}{l}\text { Expone el } \\
\text { objetivo, } \\
\text { desarrollo } \quad \mathrm{y} \\
\text { conclusiones } \\
\text { finales con gran } \\
\text { eficacia }\end{array}$ & $\begin{array}{l}\text { Expone } \quad \text { el } \\
\text { objetivo, } \\
\text { desarrollo y/o } \\
\text { conclusiones } \\
\text { finales }\end{array}$ & $\begin{array}{l}\text { Expone sólo } \\
\text { alguna parte del } \\
\text { proyecto }\end{array}$ & $\begin{array}{lr}\text { Poco adecuado o } \\
\text { no refleja } \\
\text { realmente } \\
\text { proyecto }\end{array}$ \\
\hline
\end{tabular}

Tabla 3. Rúbrica para profesores

\begin{tabular}{|c|c|c|c|c|}
\hline Competenci & Excelente (4) & Bueno (3) & Regular (2) & Malo (1) \\
\hline \begin{tabular}{l}
\multicolumn{2}{l}{ Presentació } \\
n de la \\
actividad \\
(Póster)
\end{tabular} & $\begin{array}{l}\text { Se ha explicado } \\
\text { correctamente y } \\
\text { con detalle los } \\
\text { pasos a seguir de } \\
\text { la actividad. } \\
(100 \%)\end{array}$ & $\begin{array}{lr}\text { Se } & \text { ha } \\
\text { explicado la } \\
\text { actividad a } \\
\text { grandes rasgos. } \\
(75 \%)\end{array}$ & $\begin{array}{l}\text { Se ha } \\
\text { explicado la } \\
\text { actividad de } \\
\text { manera escueta } \\
\text { y omitiendo } \\
\text { ciertas } \\
\text { informaciones. } \\
(50 \%)\end{array}$ & $\begin{array}{ll}\text { No se ha } \\
\text { explicado la } \\
\text { actividad con la } \\
\text { suficiente } \\
\text { claridad para su } \\
\text { correcta } \\
\text { realización } \\
(25 \%)\end{array}$ \\
\hline
\end{tabular}


Jesús Alba Fernández, Constantino Torregrosa Cabanilles, Romina Del Rey Tormos

\begin{tabular}{|c|c|c|c|c|}
\hline $\begin{array}{l}\text { Presentació } \\
\text { n oral }\end{array}$ & $\begin{array}{l}\text { Explicación clara } \\
\text { y concisa de la } \\
\text { tarea a llevar a } \\
\text { cabo.(100\%) }\end{array}$ & $\begin{array}{l}\text { Explicación de } \\
\text { la actividad } \\
\text { correcta } \\
\text { aunque sin } \\
\text { entrar en } \\
\text { detalles.(75\%) }\end{array}$ & $\begin{array}{l}\text { Explicación de } \\
\text { la actividad } \\
\text { con omisión } \\
\text { información } \\
\text { necesaria para } \\
\text { llevar a cabo la } \\
\text { misma.(50\%) }\end{array}$ & $\begin{array}{l}\text { Explicación } \\
\text { superficial } \\
\text { insuficiente de la } \\
\text { actividad } \\
\text { realizar. (25\%) }\end{array}$ \\
\hline $\begin{array}{ll}\text { Proceso de } \\
\text { trabajo }\end{array}$ & $\begin{array}{l}\text { El alumno ha } \\
\text { seguido correcta } y \\
\text { ordenadamente los } \\
\text { pasos fijados con } \\
\text { anterioridad.(100 } \\
\%)\end{array}$ & $\begin{array}{l}\text { El alumno ha } \\
\text { seguido los } \\
\text { pasos } \\
\text { propuestos } \\
\text { aunque no de } \\
\text { manera } \\
\text { ordenada.(75\% } \\
\text { ) }\end{array}$ & $\begin{array}{l}\text { El alumno ha } \\
\text { realizado } \\
\text { parcialmente el } \\
\text { proceso } \\
\text { propuesto con } \\
\text { anterioridad. } \\
(50 \%)\end{array}$ & $\begin{array}{l}\text { El alumno ha } \\
\text { omitido la } \\
\text { mayoría de los } \\
\text { pasos propuestos } \\
\text { en la } \\
\text { actividad.(25\%) }\end{array}$ \\
\hline $\begin{array}{l}\text { Valoración } \\
\text { general de } \\
\text { los } \\
\text { contenidos y } \\
\text { objetivos } \\
\text { adquiridos }\end{array}$ & $\begin{array}{l}\text { El alumno ha } \\
\text { alcanzado todos } \\
\text { los contenidos y } \\
\text { objetivos } \\
\text { propuestos.(100\%) }\end{array}$ & $\begin{array}{l}\text { El alumno ha } \\
\text { alcanzado la } \\
\text { mayoría de los } \\
\text { contenidos y } \\
\text { objetivos } \\
\text { propuestos.(75 } \\
\%)\end{array}$ & $\begin{array}{l}\text { El alumno ha } \\
\text { alcanzado los } \\
\text { objetivos y } \\
\text { contenidos } \\
\text { propuestos } \\
\text { parcialmente. } \\
(50 \%)\end{array}$ & $\begin{array}{lr}\text { El alumno } \\
\text { apenas } \\
\text { alcanzado ha } \\
\text { contenidos } \\
\text { objetivos } \\
\text { propuestos.(25\% } \\
\text { ) }\end{array}$ \\
\hline
\end{tabular}

\section{Resultados}

En primer lugar destacar que del total de alumnos que están siguiendo la asignatura, el 71\% han pedido realizar el trabajo voluntario. Ese $71 \%$ se han repartido en 5 proyectos distintos, 3 de ellos propuestos por los propios alumnos. Ese $71 \%$ se dividen en 8 grupos de trabajo.

Los 8 grupos realizan la exposición y presentación de póster el pasado 28 de mayo de 2015. Curiosamente, los alumnos se convierten en profesores intentando justificar qué fundamentos físicos están dentro de su proyecto.Todos los alumnos, tanto los que hacen el trabajo como los que no, rellenan la rúbrica. Además se les pide una nota global de evaluación del proyecto. A continuación se muestran dos ejemplos de dos grupos distintos (figura 1 y figura 2). Los alumnos deciden de forma global que el primer grupo tiene un 8,7 y el segundo (grupo 8) un 8,2. 
Aprendizaje basado en proyectos: Primera experiencia en la asignatura de Física del Grado en Ingeniería de Telecomunicación, Sonido e Imagen

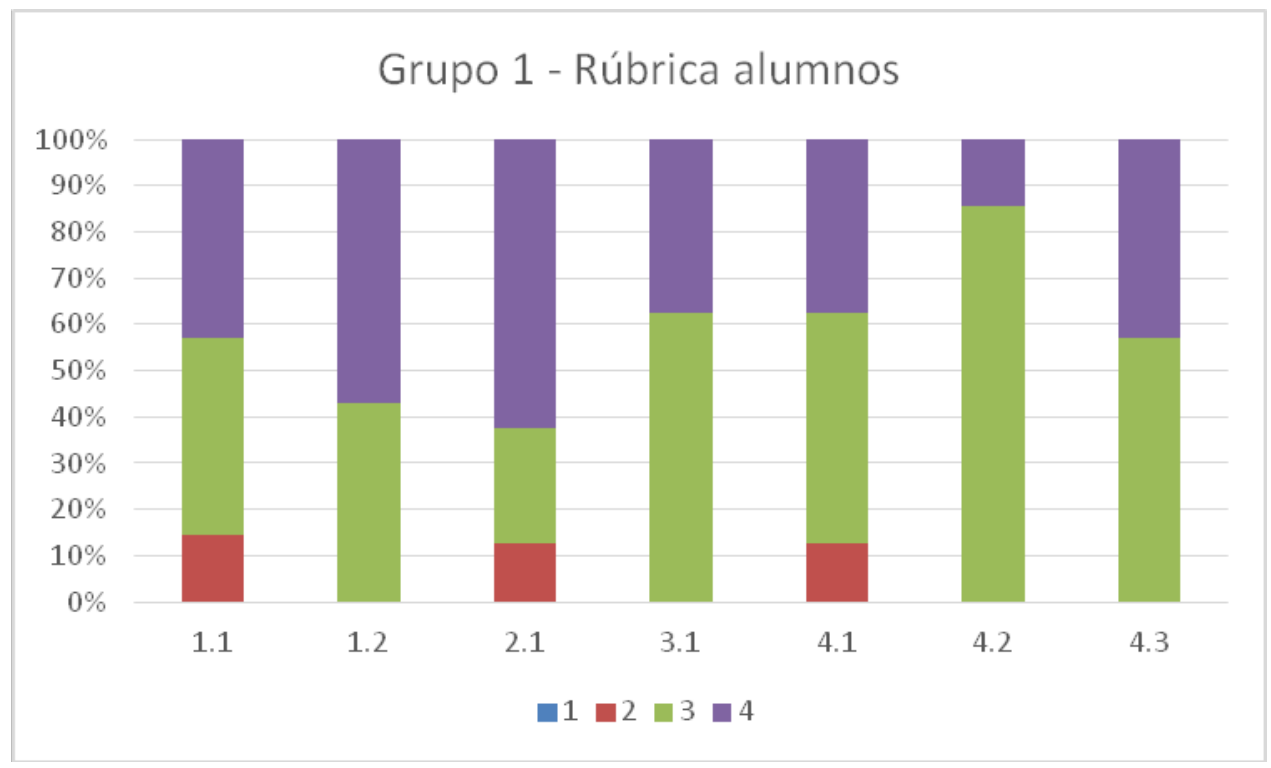

Fig. 1 Resultados de la rúbrica de alumnos grupo 1

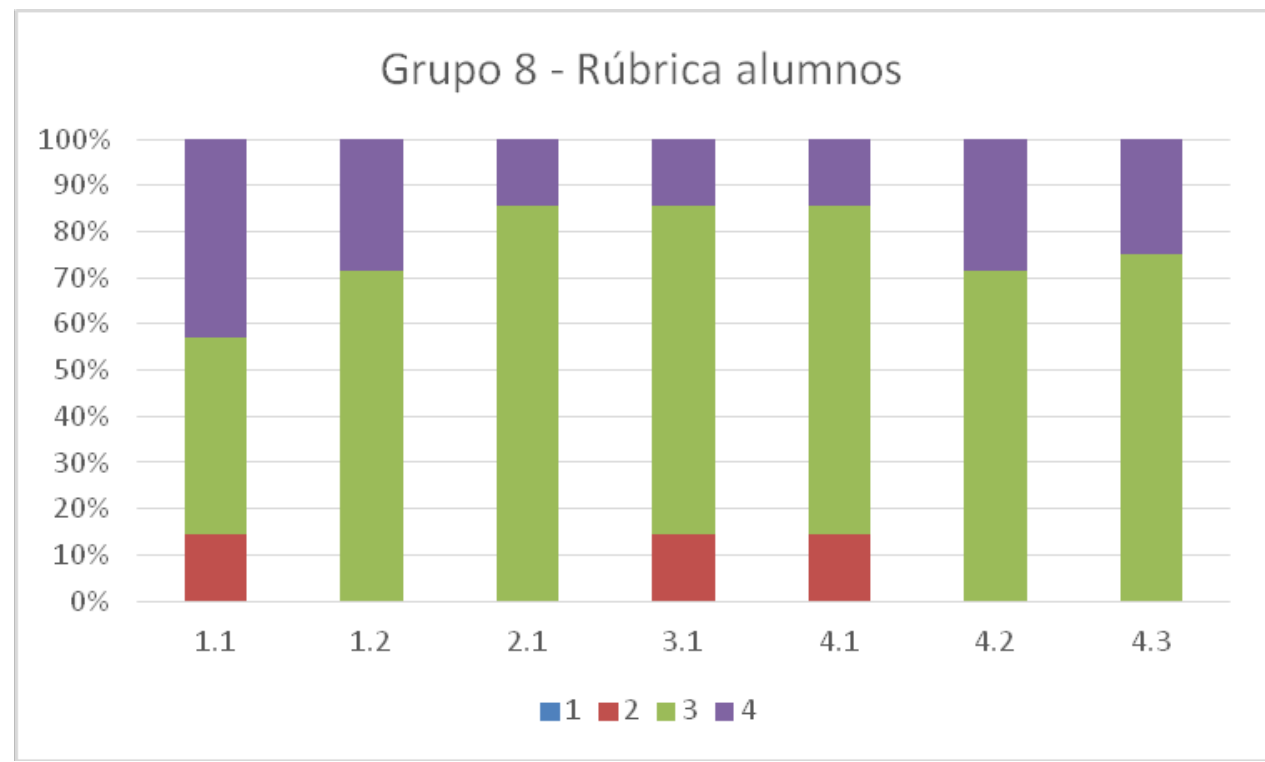

Fig. 2 Resultados de la rúbrica de alumnos grupo 8

Todos los alumnos presentan poster resumiendo su trabajo. Del mismo modo, existe una variedad grande. Desde versiones que incorporan hasta el código QR con un enlace al trabajo, hasta versiones muy básicas. 
A los grupos también se les propuso de forma voluntaria que hiciesen un video donde se pueda ver el trabajo realizado. De los 8 grupos, 4 de ellos presentan el video. Un ejemplo muy bueno que ilustra la capacidad que pueden tener los alumnos se encuentra en el enlace siguiente: https://www.youtube.com/watch?v=zz7oF8H5MsU.

Como curiosidad, se ha dado incluso el caso de que a un grupo no le funcionaba el proyecto y el resto de grupos ayudar a arreglarlo "in situ” y sobre la marcha.

Respecto a la rúbrica de profesores, lo que destaca es la diferencia de opinión entre lo que evalúan los alumnos y los profesores.

\section{Conclusiones}

En este trabajo se muestra cómo se ha conseguido motivar de forma voluntaria a través de PBLs a los alumnos del grupo de Física del GISTSI. La extrategia ha sido ofertar los trabajos de forma voluntaria y que cada grupo decida su proyecto de una cartera de proyectos o por iniciativa propia. Con esto se ha conseguido que 3 de cada 4 alumnos entre en este dinámica de forma muy activa, cambiando la percepción que tienen de la asignatura.

Respecto a resultados concretos de la evaluación. Las figuras 1 y 2 ilustran cómo están evaluando los alumnos. La percepción es que siempre se mueven en los valores 3 y 4, y cuando el punto concreto lo ven "flojo" deciden bajar al 2. Esto elimina la opción 1. Si se comparan las figuras 1 y 2 puede ver cómo la evolución en la figura 1 tiende a la máxima puntuación y la figura 2 en torno al 3. Aunque las puntuaciones son altas, se evidencia cómo el trabajo del grupo 1 ha gustado más que el del grupo 8 .

Para la realización de este proyecto, la carga de trabajo de los alumnos claramente ha aumentado. Pero también ha aumentado, y mucho, las horas que los profesores han dedicado a esta prueba. La estimación es de haber multiplicado por 3 las horas de dedicación respecto a la extrategia convencional.

\section{Agradecimientos}

Los autores agradecen al Vicerrectorado de Estudios, Calidad y Acreditación y al Instituto de Ciencias de la Educación de la Universidat Politècnica de València la concesión del Proyecto de Innovación y Mejora Educativa en la convocatoria 2014 "A02/14 Metodologías activas en aprendizaje multidisciplinar. Protocolo de creación de catálogos o mapas de motivación de título" que permite tener financiación para la presentación de este trabajo.

\section{Referencias}

Alba J., Del Rey, R., Vidal A., Roig B. Aprendizaje Basado en Proyectos en el Grado en Ingeniería de Sistemas de Telecomunicaciones, Sonido e Imagen. Caso práctico del electroscópio como experiencia interdisciplinar entre Física y Matemáticas. 23 Congreso 
Aprendizaje basado en proyectos: Primera experiencia en la asignatura de Física del Grado en Ingeniería de Telecomunicación, Sonido e Imagen

Universitario de Innovación Educativa de las Enseñanzas Técnicas (XXIII CUIEET). Valencia 15-17 de julio de 2015.

Aparicio, F., González, R. M. y Sobrevila, M. A. (2005). Formación de Ingenieros. Objetivos, métodos y estrategias. Instituto de Ciencias de la Educación, UPM.

Case, J. M. y Light, G. (2011). Emerging Methodologies in Engineering Education Research. Journal of Engineering Education, 100 (1), 186-210.

Calvo, I., López-Guede, J.M. y Zulueta, E. (2010). Aplicando la metodología Project Based Learning en la docencia de Ingeniería Técnica en Informática de Gestión, Revista de Formación e Innovación Educativa Universitaria. Vol. 3, № 4, 166-181

De Miguel, M. (2006). Modalidades de Enseñanza centradas en el desarrollo de competencias. Univer-sidad de Oviedo.

González-Jorge, H., Roca, D., Torres, S., Armesto, J. y Puente, I. (2014) Una experiencia de Aprendi-zaje Basado en Proyectos en el ámbito tecnológico: Diseño de un sistema de navegación indoor de bajo coste, Revista de Formación e Innovación Educativa Universitaria. Vol. 7, $\mathrm{N}^{\circ}$ 1, 8-19

Gregori, V. y Roig, B. (2015). Errores, optimización y resolución numérica de sistemas.Universitat Politècnica de València.

Rogawski, J. (2012). Cálculo varias variables. Reverté.

Serway, R. A. y Jewett, J. W. (2005). Física para ciencias e ingeniería. Volumen 1. México D.F. Inter-national Thomson, $6^{\mathrm{a}}$ ed.

Serway, R. A. y Jewett, J. W. (2008). Física para ciencias e ingenierías. Volumen II. México D.F. : Cengage Learning.

Tipler, P. A. (1992) Física Tomo 1. Barcelona etc. : Reverté

Tipler, P. A. (1994) Física Tomo 2. Barcelona etc. : Reverté

Urraza Digón, G. y Ortega Arceo, J.M. (2009) Diseño de una experiencia de aprendizaje por proyectos en la asignatura de Expresión gráfica y diseño asistido por ordenador mediante grupos cooperativos, Revista de Formación e Innovación Educativa Universitaria. Vol. 2, No 3, 128-138

Wright, P. H. y Samaniego, Á. H. F. (2004). Introducción a la Ingeniería. Limusa Wiley. 